\title{
Slippery Lubricant-Infused Surfaces: Properties and Emerging Applications
}

\author{
Junsheng Li, Erica Ueda, Dorothea Paulssen, and Pavel A. Levkin*
}

Bioinspired lubricant-infused surfaces exhibit various unique properties attributed to their liquid-like and molecularly smooth nature. Excellent liquid repellency and "slippery" properties, self-healing, antiicing, anticorrosion characteristics, enhanced heat transfer, antibiofouling, and cell-repellent properties have been already demonstrated. This progress report highlights some of the recent developments in this rapidly growing area, focusing on properties of lubricant-infused surfaces, and their emerging applications as well as some future challenges.

\section{Introduction}

Through the evolution and natural selection, many living organisms acquired complex solid structures on their surfaces that exhibit different outstanding properties..$^{[1]}$ Mimicking these natural structures has led to the development of different artificial advanced functional materials and surfaces in recent years. ${ }^{[2]}$ Superhydrophobicity is one example of such a biomimetic property resulting from nano- and microstructures present on natural surfaces such as the Lotus leaf, ${ }^{[3]}$ water strider's leg, ${ }^{[4]}$ rose petal, ${ }^{[5]}$ the springtail cuticle, ${ }^{[6]}$ and butterfly wing. ${ }^{[7]}$ Despite their ubiquity in research labs, superhydrophobic surfaces possess multiple weaknesses, such as repellency only toward liquids with high surface tension, low mechanical stability, low transparency, weak pressure stability, and short-term underwater stability due to the irreversible Cassie-to-Wenzel transition. Superoleophobic surfaces only solve the problem of the application to low surface tension liquids but share all the other drawbacks with superhydrophobic

\section{Dr. J. Li}

School of Chemistry

Chemical Engineering and Life Sciences

Wuhan University of Technology

Wuhan, Hubei 430070, China

Dr. J. Li, Dr. E. Ueda, D. Paulssen, Dr. P. A. Levkin

Institute of Toxicology and Genetics

Karlsruhe Institute of Technology

76344, Eggenstein-Leopoldshafen, Germany

E-mail: levkin@kit.edu

Dr. P. A. Levkin

Institute of Organic Chemistry

Karlsruhe Institute of Technology coatings. ${ }^{[8]}$ In order to solve the abovementioned limitations of traditional superhydrophobic and superoleophobic surfaces, Quéré, Aizenberg, and Varanasi proposed bioinspired slippery liquidinfused porous surface (SLIPS) or lubricant-impregnated surfaces combining the mechanical stability of a solid substrate with the liquid-like properties and molecularly smoothness of the lubricant interface. ${ }^{[9 a-d]}$ It should be noted that stabilization of a lubricant layer on solid surfaces by forming a thin polymer film that can be swollen in the lubricant was introduced in 1988 by Karakelle and Zdrahala from Bekton, Dickinson and Company. ${ }^{[9]}$ They also demonstrated excellent long-term stability, liquid repellency, and proposed various medical applications of lubricant-impregnated surfaces. SLIPSs mimic the Nepenthes pitcher plant surface, ${ }^{[10]}$ which is textured and can be lubricated with an aqueous solution. ${ }^{[11]}$ The unique slippery character of the ${ }^{[9 a]}$ Nepenthes pitcher plant's surface resulting from water lubrication helps it capture insects sliding into its interior. To mimic the Nepenthes pitcher plant surface, a lubricating hydrophobic liquid can be infused into different hydrophobic porous or rough substrates to form SLIPS. ${ }^{[9]}$ By matching the surface energy of the underlying porous substrate and the hydrophobic lubricant, the liquid can be stabilized on the surface of the substrate to form SLIPS. ${ }^{[9]}$

Depending on the impregnated lubricant, SLIPSs possess excellent repellency and drop mobility to a broad range of liquids including low surface tension liquids, and complex fluids such as blood or cell medium. ${ }^{\left[{ }^{9 a}\right]}$ This liquid repellency as well as SLIPS' self-healing properties are due to the mobility of the lubricant trapped inside the porous surface structures. ${ }^{[12]}$ Lubricant-infused surfaces demonstrated icephobic, stainresistant, biofilm- or cell-repellent or marine antibiofouling properties as well as tolerance to high pressure and transparency. ${ }^{[9,13]}$ All of these unique properties make SLIPS interesting for the development of novel functional materials and various practical applications.

The goal of this progress report is not to comprehensively review this broad and dynamic research field but rather highlight selected important aspects that have been less reviewed so far despite their potential. Some important and related papers could not be cited due to the length limitations. We also refer readers to several excellent reviews covering applications of SLIPS for heat transfer and condensation, ${ }^{[14]}$ antibiofouling properties, ${ }^{[15]}$ antiicing properties ${ }^{[16]}$ as well as medically relevant applications. ${ }^{[17]}$ 


\section{Fabrication}

To prepare thermodynamically stable SLIPS, the following criteria should be fulfilled: (a) the impregnated lubricant (liquid A) should be nonreactive to the substrate and to the repelled liquid (liquid B); (b) liquid A and B are immiscible; (c) the substrate should be preferentially wetted by the infused liquid $A$ rather than liquid $B$ and (d) the substrate should be rough, porous or swellable in the lubricant. ${ }^{[9 a, 18]}$ Detailed thermodynamic conditions required for the formation of stable SLIPS have been described elsewhere. ${ }^{[9 a, 18]}$ For example, Preston et al. ${ }^{[18]}$ developed a model that predicts interfacial surface energies to determine whether an arbitrary combination of solid and lubricant will repel a given impinging fluid. The same authors demonstrated stable SLIPS of a polar lubricant on uncoated high-surface energy solids, which enabled repulsion of discrete droplets of very low-surface-tension fluids (e.g., butane, $\gamma=13 \mathrm{mN} \mathrm{m}^{-1}$ ). ${ }^{[18]}$ In addition, lubricant drainage caused by the "cloaking effect," that is formation of a thin lubricant film on the impinging droplet, should be minimized to enable long-term stability. ${ }^{[18,19]}$

One of the main advantages of SLIPSs is the simplicity of their fabrication, usually performed by simple impregnation of a lubricant into an underlying substrate (Figure 1A). To stabilize the lubricant layer, the substrate should be porous, rough, or swellable by the lubricant. Various porous substrates have been utilized for this purpose ${ }^{[20-30]}$ (Figure 1B), while the use of flat polymers swollen in the lubricant was introduced more recently ${ }^{[31]}$ with PDMS/silicone used most often. ${ }^{[32,33]}$ For example, Eifert et al. demonstrated that a stable SLIPS could be created on various surfaces by impregnating a thin layer of PDMS attached to the surface with a silicone oil. ${ }^{[31]} 2 \mathrm{D}$ rough surfaces are relatively easy to create but their long-term stability is limited due to the finite amount of a lubricant trapped in the rough surface compared to the bulk 3D porous and swellable materials.

SLIPS fabrication on industrially relevant surfaces is of great interest for practical applications, especially as a nonstick coating for viscous fluids, oils, paints, etc. ${ }^{[34-36]}$ Thus, Aizenberg and co-workers created SLIPS on aluminum surfaces via the electrochemical deposition of polypyrrole, followed by Krytox 100 infusion. ${ }^{[37]}$ SLIPS fabrication on a copper surface by treating the surface in a hot $\left(96^{\circ} \mathrm{C}\right)$ alkaline solution, surface modification with (tridecafluoro-1,1,2,2,tetrahydrooctyl)-1-trichlorosilane, followed by Krytox infusion, was also reported. ${ }^{[38]}$ SLIPSs were also created on steel surface with an electrodeposited nanoporous tungsten oxide stabilization layer. ${ }^{[39]}$ Moreover, several other groups have reported that SLIPS could be used as an anticorrosive coating on various metals. ${ }^{[40,41]}$ Even a paper-based SLIPS was demonstrated by functionalizing filter paper with a hydrophobic silane and infusing it with perfluoropolyether. ${ }^{[42]}$ Such paper SLIPS is interesting because it combines mechanical properties of paper with the excellent liquid repellency of SLIPS and can be easily folded into functional shapes. Likewise, Guo et al. functionalized nanofibrillated cellulose films by coating them through polycondensation of trichlorovinylsilane. ${ }^{[25]}$ Modification with perfluoroalkyl thiols resulted in robust superhydrophobic surfaces, which could then be further
Erica Ueda obtained a B.S. in Mechanical Engineering from UCLA in 2006 and a M.S. in Mechanical Engineering from MIT in 2008. She is a Ph.D. student in the Faculty of Chemistry and Biosciences at the Karlsruhe Institute of Technology. Her research is focused on developing cell screening platforms such as cell, droplet, and hydrogel microarrays.

\section{Dorothea Paulssen obtained a B.Sc in Biochemistry and Cell Biology from Jacobs University Bremen in 2008, as well as a M.Sc. in Systems Biology from ETH Zurich and a M.Sc. in Drug Design from UCL. She is a Ph.D. student in the group of Dr. Levkin in the Institute of Toxicology and Genetics at the Karlsruhe Institute of Technology. Her research is focused on SLIPS and the development of read-out strategies for droplet microarray platforms.}

\section{Pavel Levkin is head of the Biofunctional Materials research group at Karlsruhe Institute of Technology (KIT), Germany. He graduated from the Institute of Fine Chemical Technology, Moscow and obtained his Ph.D. in Organic Chemistry from the University of Tübingen in Germany, fol- lowed by postdoctoral work at the University of California, Berkeley. Pavel Levkin is the cofounder of ScreenFect $\mathrm{GmbH}$} and Aquarray $\mathrm{GmbH}$. His research focuses on the development of functional and responsive materials, and surfaces for biomedical and biotechnological applications.

transformed into transparent SLIPS. Various other methods for creating SLIPS coatings on different substrates were reported including for example texturing of surfaces by, e.g., femtosecond laser etching and subsequent silanization, ${ }^{[26]}$ layer-by-layer techniques, ${ }^{[38]}$ as well as nanoparticle coatings ${ }^{[43]}$ - to name a few.

\section{Stability}

SLIPS relies on the uppermost mobile lubricant layer as opposed to the trapped air pockets in the case of 
(A)

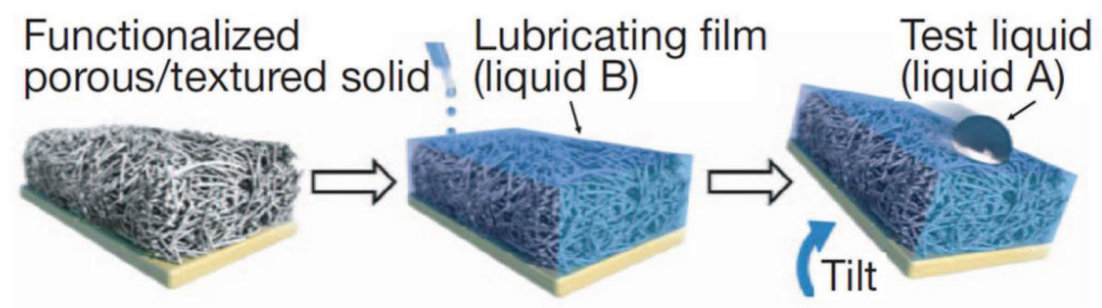

(B)
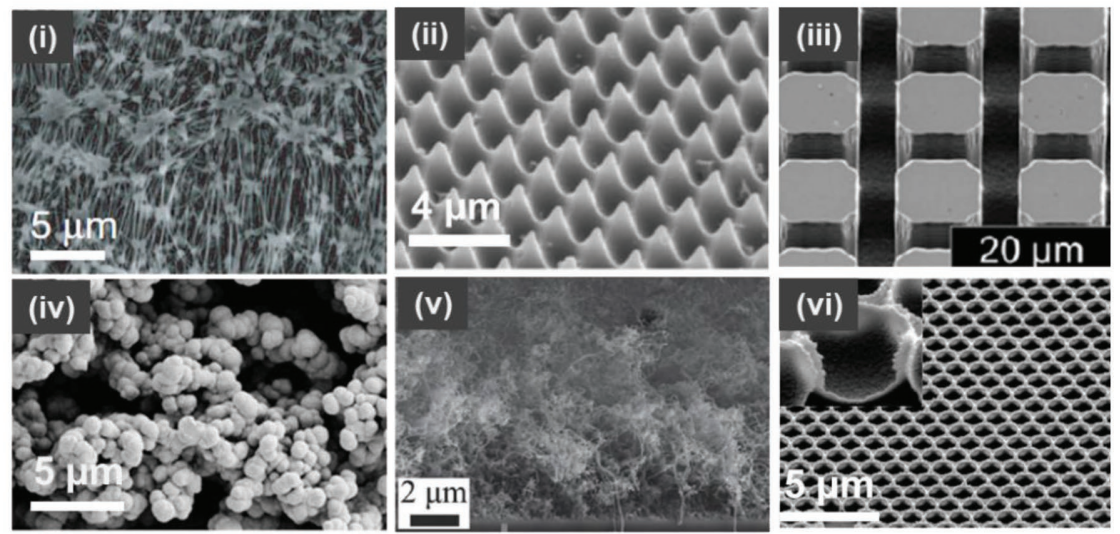

Figure 1. A) Scheme showing the fabrication of a lubricant-infused surface. Reproduced with permission. ${ }^{[9 a]}$ Copyright 2011 , Springer Nature. B) SEM images of representative porous substrates used to fabricate SLIPS: (i) porous Teflon. Reproduced with permission. ${ }^{9 a}$ ] Copyright 2011 , Springer Nature. (ii) Ordered polyacrylate. Reproduced with permission. ${ }^{[9 a]}$ Copyright 2011 , Springer Nature. (iii) Silicon nanoarray. Reproduced with permission. ${ }^{[29]}$ Copyright 2013, American Chemical Society. (iv) porous poly(butyl methacrylate-co-ethyleneglycol dimethacrylate). Reproduced with permission. ${ }^{[22]}$ Copyright 2013 American Chemical Society. (v) porous silicone nanofilament, Reproduced with permission. ${ }^{[30]}$ Copyright 2014 , Wiley-VCH. (vi) inverse colloidal monolayer. Reproduced with permission. ${ }^{[28]}$ Copyright 2013, the Authors, published by Springer Nature.

superhydrophobic surfaces. This makes SLIPS more pressure stable and solves the problem of the Cassie-to-Wenzel transition of superhydrophobic surfaces. The lubricant layer, however, may be depleted leading to the loss of the "slippery" characteristics of SLIPS. ${ }^{[4]}$ Another specific problem of SLIPS is the droplet cloaking effect which can be only overcome by choosing appropriate lubricants. ${ }^{[9 \mathrm{~d}, 19]}$

The first approach to increase SLIPS' stability is to exploit the physical interaction between the lubricant and the porous substrate. The effect of the length scale and hierarchy of the surface topography on the stability of the lubricant impregnating the rough surface was systematically investigated using perfluoropolyether as a lubricant. ${ }^{[45]}$ The stability of SLIPS was investigated by measuring water contact angles before and after spinning of the substrate. For the microtextured and hierarchically textured surfaces, the lubricant layer was reduced by shear forces, reducing its liquid repellency reflected in the increase of ethanol and water contact angle hystereses. However, SLIPS based on substrates with uniform nanostructures exhibited higher stability under strong shear forces. Highly robust SLIPS were prepared using a series of porous substrates with uniform nanopores ranging from 140 to $1060 \mathrm{~nm}$ (Figure 1B-vi).[28] The liquid-repellent property of this SLIPS remained after mechanical damage or after more than 9 months vertical storage.

Covalent immobilization of the lubricant to the underlying substrate may also increase SLIPS' stability. In addition, lubricant immobilization reduces the importance of surface topography for the lubricant stabilization, making even flat nonporous substrates appropriate for the coating. ${ }^{[31]}$ Surface lubrication and lubricant layer stabilization have been very active fields for several decades. ${ }^{[9 \mathrm{e}]} \mathrm{A}$ recent example to be highlighted includes Shiratori and co-workers, who developed a robust SLIPS with the lubricant covalently bonded to the underlying LBL substrate. ${ }^{[46]}$

Another design principle aimed at the increase of longterm stability of SLIPS is based on the regeneration of the lubricated film through the self-secretion or resupply of the lubricant by "vascularization" of the material. Thus, Howell et al. introduced a 3D "vascular system" into a PDMS substrate functioning as a lubricant reservoir to replenish lubricant. ${ }^{[47]}$ The rapid replenishment of a lubricating liquid by injecting the liquid into a porous substrate with internal channel networks was also shown to prolong the lifetime of SLIPS. ${ }^{[48]}$ Another modification of such self-secreting SLIPS was prepared by casting a mixture comprised of a copolymer of urea and polydimethylsiloxane, silicone oil, and THF. ${ }^{[49]}$ The resulting SLIPS contained lubricant droplets incorporated into the bulk polymer layer, which could release lubricant upon mechanical damage. Alternatively, a sandwiched configuration of SLIPS, containing a middle lubricant layer, which could self-secrete lubricant to the surface once the surface lubricant layer is depleted, was introduced to enhance the surface stability. ${ }^{[50]}$ 
The evaporation of lubricant can lead to SLIPS' fast degradation and loss of its slippery character. Increase of molecular weight, cross-linking of the lubricant, or use of ionic liquids ${ }^{[1,52]}$ can reduce lubricant volatility. This is especially important for high-temperature or high-vacuum applications. Thus, SLIPS formed with 1-ethyl-3 methylimidazoliumbis(trifluoromethylsu lfonyl)imide as an extremely nonvolatile lubricant, could withstand $12 \mathrm{~h}$ of heating at $250^{\circ} \mathrm{C}$ or incubation in a chamber at a pressure of $0.7 \mathrm{~Pa}$ for 24 h. ${ }^{[51]}$ Notably, Greeson et al. employed a paramagnetic ionic liquid to fabricate SLIPS - the thermal stability of which could be further enhanced with applied external magnetic field. ${ }^{[53]}$

\section{Stimuli-Responsive SLIPS}

Incorporation of responsive components into lubricant-infused surfaces can lead to various interesting dynamic, adaptive and responsive properties. Both lubricant and solid components of SLIPS can be used to achieve the desired dynamics or responsiveness of the surface, which makes it also more flexible in comparison to one-component solid interfaces. Examples of potential stimuli include but are not exclusive to temperature, magnetic field, electric field, mechanical stimuli, $\mathrm{pH}$, or light. Some examples of responsive SLIPS are given below.

By using a solidifiable liquid (paraffin) as the lubricant, thermal responsiveness was introduced into SLIPS. ${ }^{[54]}$ Once temperature was lowered below a critical temperature, such SLIPS lost its slippery characteristics, which could be restored by increasing temperature. In a similar example, the slippery property could be "activated" or "deactivated" by melting or freezing paraffin-based lubricant in an organogel. ${ }^{[55]}$ Melted $n$-tetracosane was impregnated into a PDMS elastomer at $150{ }^{\circ} \mathrm{C}$ to form an organogel film. At room temperature, the organogel film showed a high water contact angle hysteresis (CAH) of $\approx 50.2^{\circ}$ due to the high surface roughness, which dropped to $1.9^{\circ}$ upon heating to $75{ }^{\circ} \mathrm{C}$. Zheng et al. introduced SLIPS based on a silicone lubricant possessing the lower critical solution temperature (LCST), which made the surface hydrophilic below LCST (i.e., $40{ }^{\circ} \mathrm{C}$ ) and hydrophobic water repellent above LCST. ${ }^{[56]}$ Transparency of the SLIPS wetted with water became also temperature dependent because of the change in the composition of the liquid layer at different temperatures.

Tian et al. prepared a SLIPS with ZnO nanorod array as the substrate and a superparamagnetic lubricant composed of silicone oil and $\mathrm{Fe}_{3} \mathrm{O}_{4}$ nanoparticles. ${ }^{[57]}$ The SLIPS showed slipperiness only in the absence of the magnetic field. Varanasi and co-workers ${ }^{[58]}$ demonstrated that a SLIPS with ferrofluid as the lubricant could cloak a water droplet placed on it, which enabled manipulation of the droplet using magnetic field (Figure 2A,B). A ferrofluid-infused porous surface with reconfigurable multiscale topography has also been reported. ${ }^{[59]}$ An applied magnetic field gradient changed the initially smooth surface of the ferrofluid into various multiscale hierarchical topographies shaped by the size, arrangement, and orientation of the confining microstructures in the magnetic field. This effect was used to self-assemble colloidal particles at the micrometer scale; to regulate flow of liquid droplets; and to achieve switchable adhesion and friction, liquid pumping and removal of biofilms at the centimeter scale.

A micropillar array containing magnetic carbonyl iron was used to form magnetically responsive SLIPS. ${ }^{[60]}$ When a magnetic field was imposed, the orientation of the micropillars self-adjusted, which resulted in the redistribution of the lubricant on the surface. By proper manipulation of the magnetic field, free switching between the slippery state and superhydrophobic state was realized. The switching enabled the control of the speed of droplet shedding on the surface. Cao et al. developed SLIPS based on a magnetically responsive solid substrate composed of a PDMS-cobalt microparticles composite. ${ }^{[61]}$ This SLIPS could respond to the magnetic field due to the change of orientation of the microcilia of the underlying substrate.

Smart manipulation of droplets using electric field could be realized by introducing anisotropy into the substrate of SLIPS. ${ }^{[62]}$ Control of droplet movement on SLIPS by electric field was also demonstrated simply by using conductive droplets, lubricants or conductive underlying porous material. ${ }^{[63,64]}$

Strain-responsive SLIPS was also reported using elastic and structured PDMS substrate coated with a lubricant, which could change its transparency and droplet mobility by simple elongation of the substrate. ${ }^{[12]}$ Substrate elongation led to the exposure of PDMS micropillars and loss of the slipperiness. The sensitivity of the responsiveness could be adjusted by the amount of lubricant present on the SLIPS. These deformation-induced changes in SLIPS were reversible, predictable and could be used to control the mobility of water droplets on the SLIPS. A similar strain-responsive SLIPS prepared by impregnation of a perfluoropolyether into the modified thermoplastic polyurethane membrane could be also used to manipulate droplet motion on the SLIPS by external force. ${ }^{[65]}$ Inspired by the fact that strain-induced deformation would result in the color change of a photonic crystal, a SLIPS with inverse opal polyurethane as the substrate was proposed as a strain responsive surface. ${ }^{[66]}$ Since the straininduced surface color change correlates with the contact angle change, this SLIPS can be used as a visually self-reporting surface.

Recently, Wooh and Butt created a slippery surface using titanium oxide nanoparticles that remained photocatalytically active after impregnation. ${ }^{[67]}$ The thin PDMS layer allowed for the photocatalytic degradation of organic contaminants by the underlying $\mathrm{TiO}_{2}$ substrate, combining "active" self-cleaning with slipperiness of this lubricant infused surface. Lightresponsive SLIPS have likewise been proposed as suitable platforms for controlled droplet manipulation. A powerful example of this was presented by Gao et al. ${ }^{[68 \mathrm{a}]}$ who created photothermal responsive SLIPS that were capable of transporting droplets along desired trajectories with controllable velocity and direction. In another approach, Wang et al. ${ }^{[68 b]}$ created SLIPS that allowed for droplet manipulation upon a synergistic stimulus of light and electricity combined.

These examples of responsive lubricant-infused surfaces show the great potential of combinations of slippery lubricant infused surfaces, being already functional, with additional 

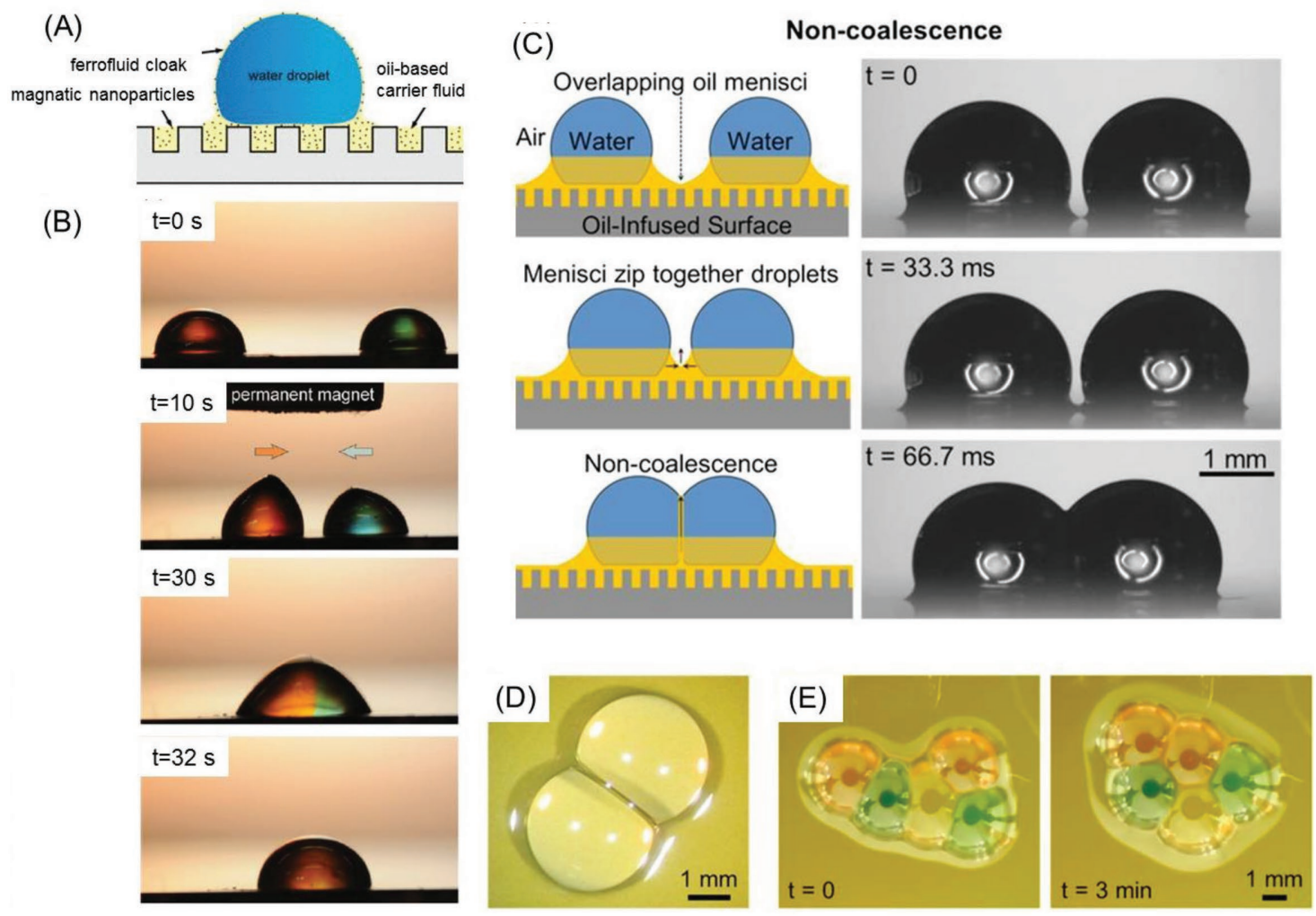

Figure 2. A) Scheme showing the cloaking of a water droplet on a SLIPS based on ferrofluid. B) Time lapse images showing the manipulation of water droplets on the SLIPS based on ferrofluid: a magnet was placed between two droplets and guided the movement and coalescence of the droplets. A,B) Reproduced with permission. ${ }^{[58]}$ Copyright 2014, AIP. C) Schematic representation (left) and images (right) showing the formation of stable oil films between two water droplets with overlapped oil menisci on SLIPS. D) Top-down pictures of the noncoalescing droplets. E) Rearrangement of the noncoalescing droplets on SLIPS to minimize the overall interfacial energy. Panels C-E reproduced with permission. ${ }^{110]}$ Copyright 2014, The Authors, Published by the National Academy of Sciences.

dynamic and responsive properties. It is clear that many more possibilities for such dynamically controlled and responsive interfaces exist and will soon be reported and inevitably be used as parts of various applications, such as integrated control systems for the manipulation and transport of liquids, thermal management, microfluidics, or fouling-release materials.

\section{Patterned SLIPS}

There is a growing interest in the development and application of surfaces patterned with different wettability. ${ }^{[69]}$ Combination of different wettability states on the same surface could lead to new surface properties, such as discontinuous dewetting, ${ }^{[70]}$ which could lead to a number of new surface related applications. Up to now, such patterned surfaces have been used for water collection, ${ }^{[71]}$ patterning of droplets, biomolecules, hydrogels and cells, ${ }^{[72]}$ manipulation of liquid flow, ${ }^{[73,74]}$ control of bioadhesion, ${ }^{[69 b, 75]}$ miniaturized separation and diagnostics. ${ }^{[76,77]}$ Superhydrophobic-hydrophilic micropatterns are often used for the aforementioned applications. ${ }^{[69 \mathrm{~b}]}$ Despite the potential of superhydrophobic-hydrophilic micropatterns, they usually can work only with aqueous solutions. Superoleophobic-oleophilic micropatterns are expected to show better solvent compatibility and their development has only recently been reported. ${ }^{[72 e, 78]}$ However, superoleophobicoleophilic micropatterns are difficult to fabricate; they suffer from low mechanical as well as long-term stability. Similarly to superhydrophobic-hydrophilic micropatterns, ${ }^{[69 b]}$ combination of the slippery lubricant-infused surfaces with other types of surfaces (hydrophilic, hydrophobic, adhesive, superhydrophobic, etc.) on the same surface in precise micropatterns is very interesting because it can lead to completely novel macroscopic properties and important new applications. We recently reported fabrication of a surface with micropatterns of slippery cell-repellent lubricant-infused and cell-adhesive hydrophilic surfaces. We demonstrated that these substrates could be used for patterning aqueous solutions and cells, ${ }^{[79]}$ including long-term maintenance of cell micropatterns (Figure 3A). Furthermore, Bruchmann et al. showed that such SLIPS patterns could be used to form arrays of biofilm microclusters and both the size and shape of the microclusters could be controlled, which is important for studying biofilm properties and usually cannot be achieved with superhydrophobic-hydrophilic micropatterns. ${ }^{[80]}$ Vogel et al. reported the development of a SLIPS pattern with an inverse monolayer substrate, which was used to pattern water, octane and even blood samples (Figure 3B). ${ }^{[28]}$ By the same principle, patterning of underlying substrate with hydrophilic material, sliding tracks have been 
(A)

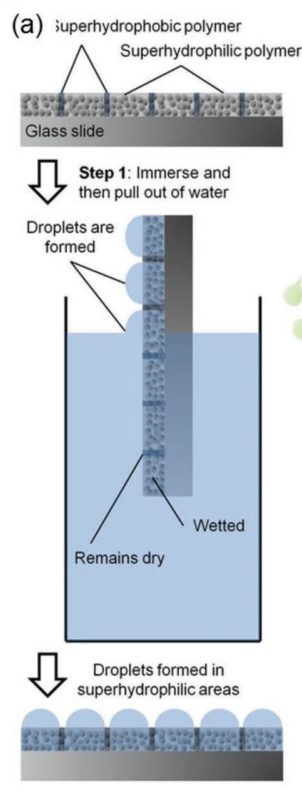

(B)
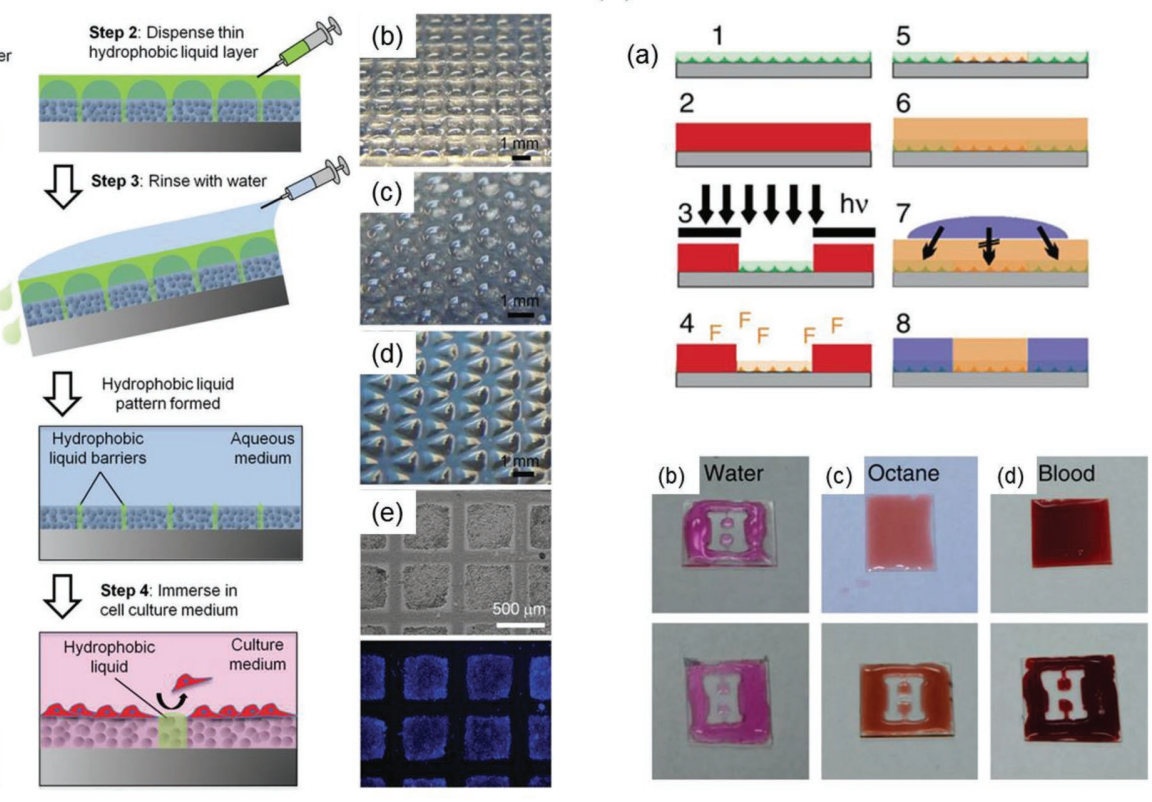

(C) $($ (a)

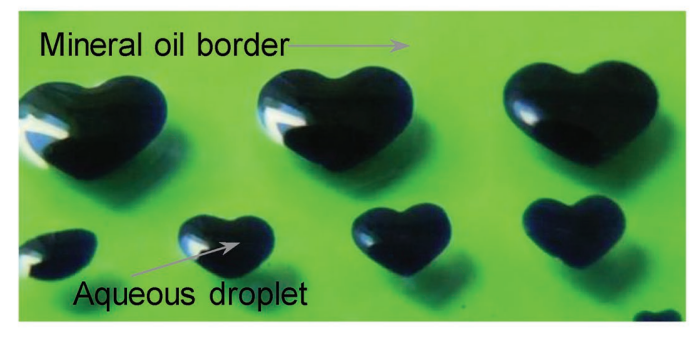

(D)

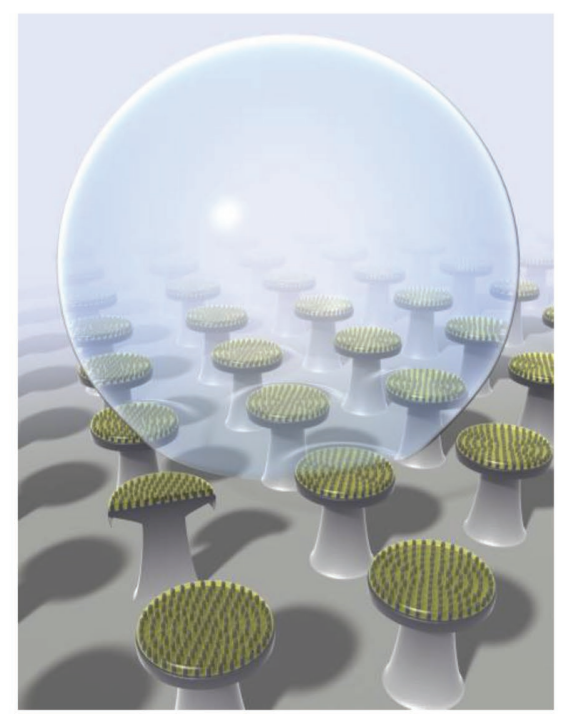

(b)

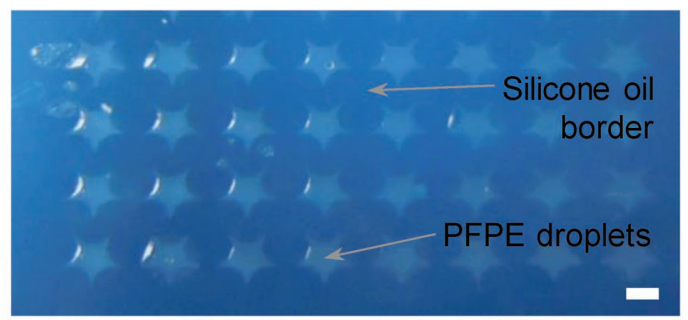

Figure 3. A) Schematic of the formation of a SLIPS pattern on a superhydrophilic nanoporous polymer surface with a superhydrophobic pattern. (b-d) Water droplets in superhydrophilic spots separated by $100 \mu \mathrm{m}$ wide lubricating liquid barriers with different array pattern geometries. (e) HEK 293 cells cultured on a SLIPS micropattern $(500 \mu \mathrm{m}$ side length square, $100 \mu \mathrm{m}$ barrier). Brightfield and DAPI channel (blue) images are shown. A) Reproduced with permission. ${ }^{[79]}$ Copyright 2013, Wiley-VCH. B) Preparation and application of a transparent SLIPS pattern. (a) An inverse monolayer substrate (1) is coated with photoresist (2), irradiated through a photomask, and developed to create the surface pattern (3). Vapor-phase fluoro silanization used to create fluorinated surface functionalities at the exposed surface regions (4); the protected surface regions remain nonfunctionalized after removal of the photoresist (5). Due to its low surface energy, the lubricant first creates a homogeneous liquid film on the surface (6). Addition of a second liquid (7) that has a higher affinity to the non-functionalized surface areas than the lubricant leads to localized replacement of the lubricant at the non-functionalized surface areas (8). Patterning of (b) rhodamine B stained water, (c) DFSB-K175 stained octane, and (d) sheep blood on the SLIPS pattern. B) Reproduced with permission. ${ }^{[28]}$ Copyright 2013, the Authors, published by Springer Nature. C) Images of patterned SLIPs with alternative lubricant system including (a) water droplets surrounded by a mineral oil lubricant and (b) perfluoropolyether droplets surrounded by a silicone oil background. C) Reproduced with pernmission. ${ }^{[83]}$ Copyright 2018, wiley-VCH. D) Schematic of the structural architecture to combine slippery surfaces with superoleophobic double-reentrant structures. Reproduced with permission. ${ }^{[11]]}$ Copyright 2018, Wiley-VCH.

realized to direct gravitational droplet movement of a range of liquids such as water and ethanol. ${ }^{[81]}$ A similar patterned V-shaped surface also allowed for the positioning and movement of droplets based on capillary interactions. ${ }^{[82]}$ Recently, we investigated the capability of different liquids to displace the lubricant from higher surface energy regions and showed that both high and low surface tension liquids can replace it, thereby forming droplets sharply following underlying surface 


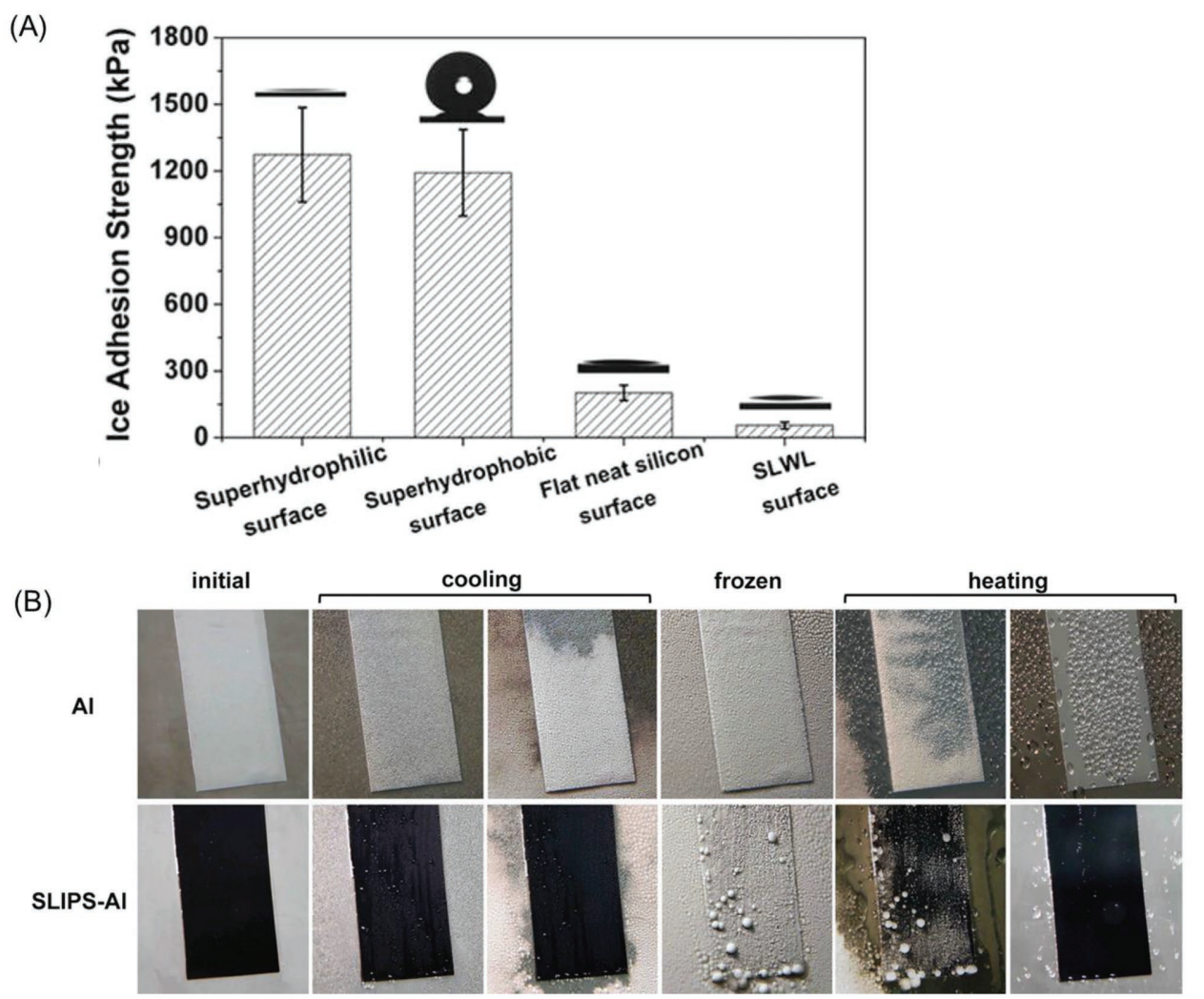

Figure 4. A) Average ice adhesion force of four different surfaces: a textured silicone surface modified with piranha solution; the same textured silicone surface modified with (heptadecafluoro-1,1,2,2-tetradecyl)-trimethoxysilane; a smooth silicon surface; SLIPS based on the same textured silicon surface. Reproduced with permission. [118] Copyright 2013, American Chemical Society. B) Snapshots extracted from movies showing ice formation by deep freezing $\left(-10^{\circ} \mathrm{C}, 60 \% \mathrm{RH}\right)$ and subsequent de-icing by heating on an aluminum substrate (top) and SLIPS-Al (bottom). The samples were fixed

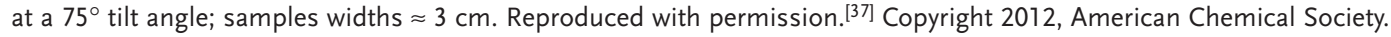

energy patterns (Figure 3C). ${ }^{[83]}$ By changing the chemical patterning from fluorinated to aliphatic groups, patterns of mineral and silicone oils could be created. This method could be used to pattern perfluoro oils into designer-shape droplets, and to demonstrate formation of 2D micropatterns of threephase liquid systems (fluorinated, organic, and aqueous), which is usually difficult to achieve without superhydrophobicsuperoleophobic-hydrophilic patterns.

Besides the chemical composition of the surface, the topography of the surface could be patterned enabling formation of SLIPS patterns based on topographical differences. Thus, Chen et al. ${ }^{[84]}$ introduced microstructured polyelectrolyte multilayer films with erasable and patternable surface topography. Upon exposure to water, the surface could be smoothened reducing the stability of a lubricant layer. Such topographically patterned lubricant-infused surfaces could be utilized for guiding the sliding direction of water droplets, controlling droplet sliding speed, or for capturing water droplets at smooth regions. ${ }^{[84]}$

\section{Anti-Icing SLIPS}

Ice accumulation on surfaces such as power lines, wind turbines, planes, road signs is a challenging problem leading to great economic losses. One of the important SLIPS' properties is their icephobicity (Figure 4). ${ }^{[37,85]}$ It was reported that water nucleation on SLIPS could be inhibited because of the reduction of nucleation sites due to SLIPS' homogeneous and ultrasmooth nature. ${ }^{[85]}$ Even if water droplets condense on SLIPS, they roll off easily from the surface due to its extremely low contact angle hysteresis. Kim et al. studied the formation of frost and ice on SLIPS formed on industrial grade aluminum (Figure 4B). ${ }^{[37]}$ Owing to the decreased water nucleation on SLIPS and the facilitated water removal from its surface, no frost formation was observed even at a high cooling rate of $2{ }^{\circ} \mathrm{C} \mathrm{min}-1$. It was also shown that the ice adhesion strength on SLIPS-coated aluminum was much lower than on untreated aluminum, making ice removal easier. Varanasi and co-workers showed ${ }^{[86]}$ that the existence of the top lubricating layer was critical for reducing the ice adhesion strength to SLIPS. Zhu et al. prepared PDMS SLIPS by blending silicon oil into the PDMS curing mixture. ${ }^{[87]}$ The ice adhesion tensile strength on this SLIPS could be reduced to $<100 \mathrm{kPa}$, a critical value below which ice removal is considered to be easy. Even lower ice-adhesion strength $(\approx 2 \mathrm{~Pa})$ was recently achieved through a slippery surface based on a ferromagnetic liquid, ${ }^{[88]}$ and an organic liquidswelled organogel $(\approx 0.4 \mathrm{kPa})$ termed SLUG (Self-LUbricating organoGel). ${ }^{[89]}$ 


\section{Cell-Repellent and Antibiofouling SLIPS}

Coatings resistant to bacterial adhesion or repellent toward mature biofilms and other microorganisms are important in applications ranging from medical implants and catheters to coatings for ship hulls and other marine infrastructure. Traditionally, antibacterial surfaces were based either on coatings with the capability of releasing antibacterial agents or various types of hydrophilic cell repellent, e.g., PEGylated coatings. Despite the progress in the development of antibacterial surfaces, surfaces with a long-term robust antibacterial or bacteria repellent effect are still difficult to achieve. Due to the liquid-like properties of SLIPS interfaces, defect-free smooth surfaces, liquid repellency, self-healing properties, they were expected to show repellency toward live microorganisms, cells, bacteria, and bacterial biofilms. Aizenberg and co-workers first demonstrated ${ }^{[90]}$ that adhesion of Pseudomonas aeruginosa, Staphylococcus aureus, and Escherichia coli biofilms on SLIPS was greatly reduced both in static conditions and in flow conditions, compared to that on Teflon or PEGlyated surfaces (Figure 5A). Our group investigated formation of biofilms of laboratory strain (PA14) and waste water isolated wild strains (PA30, PA910, and PA49) of P. aeruginosa on SLIPS. ${ }^{[91]} 7 \mathrm{~d}$ bacterial culture experiments performed in a flow bioreactor revealed good resistance of SLIPS against
P. aeruginosa (Figure 5B). However, the antibiotic multiresistant $P$. aeruginosa isolate PA49 was able to form dense biofilms on the SLIPS studied. Bacterial resistance of SLIPS against other bacteria strains, i.e., isolated environmental Desulfovibrio sp., as well as E. coli and S. aureus strains, was also shown. ${ }^{[92]}$ However, Wilson et al. analyzed the antibacterial property of slippery lubricant-infused porous polymethacrylate surfaces in a drip flow reactor. The results showed that $P$. aeruginosa (PA14) had a greater amount of biofilm growth on the SLIPS in comparison to the glass surfaces, which contradicted prior reported results. This work reinforces the strain-dependent bacterial adhesion behavior and the need for further investigations. ${ }^{[93]}$

While conventional SLIPS could successfully inhibit the adhesion of biofilm on the surface, development of active SLIPS that not only inhibits bacterial growth on the surface but also releases specific bioactive molecules can be beneficial in practical applications. ${ }^{[94]}$ Manna et al. showed that sustained release of antimicrobial agent from SLIPS could be realized by incorporation of an antimicrobial agent to the porous underlying surfaces prior to lubricant infusion. ${ }^{[94 \mathrm{~b}]}$ The active SLIPS demonstrated long-term inhibition of bacterial growth both on the surface and in the culture media.

Due to its excellent antibiofouling performance, SLIPS have been tested in vivo as coatings for implants, ${ }^{[95]}$ endoscopes, ${ }^{[96]}$ and catheter surfaces. ${ }^{[97]}$ Excellent antibiofouling performance
(A)
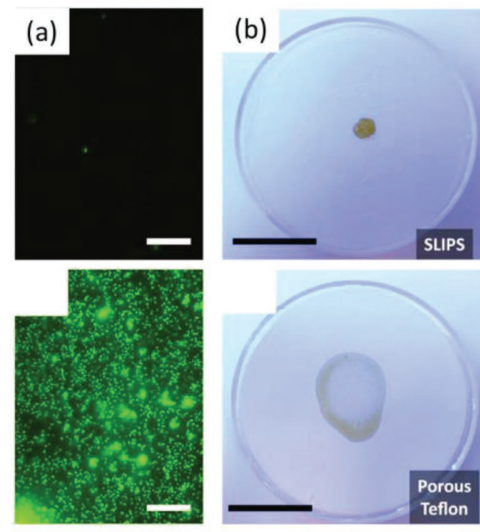

(B)

\begin{tabular}{lccc} 
& \multicolumn{3}{c}{ surface coverage in \% } \\
\cline { 2 - 4 } & $\begin{array}{c}\text { slippery BMA- } \\
\text { bacterial type }\end{array}$ & $\begin{array}{c}\text { glass slide } \\
\text { EDMA surface }\end{array}$ & $\begin{array}{c}\text { BMA-EDMA } \\
\text { surface }\end{array}$ \\
P. aeruginosa PA14 & $0.92 \pm 0.33$ & $2.14 \pm 0.5$ & $2.43 \pm 0.89$ \\
P. aeruginosa PA30 & $1.95 \pm 1.19$ & $2.45 \pm 0.16$ & $86.27 \pm 4.52$ \\
P. aeruginosa PA910 & $0.87 \pm 0.25$ & $2.28 \pm 0.37$ & $1.52 \pm 0.15$ \\
P. aeruginosa PA49 & $11.96 \pm 6.27$ & $6.45 \pm 1.87$ & $36.34 \pm 14.21$ \\
a $1: 4$ BHI broth, flow rate $0.94 \mathrm{~mL} \mathrm{~min}^{-1}$. &
\end{tabular}

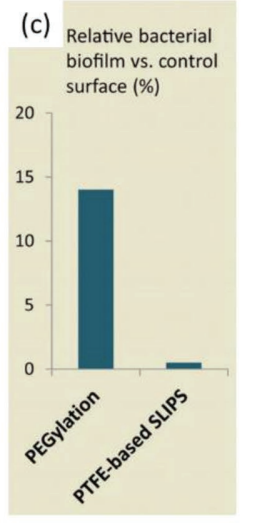

(D)

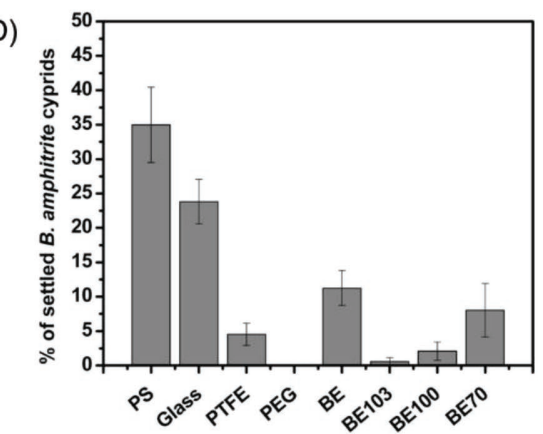

Figure 5. A) Biofilm attachment on SLIPS. (a) Fluorescence microscope images of $P$. aeruginosa (PA 14) after $48 \mathrm{~h}$ of culture on SLIPS prepared with superhydrophobic PTFE (top) and on superhydrophobic PTFE (bottom), scale bar $300 \mu \mathrm{m}$. (b) Image of the remains after evaporation of a drop of PA 14 biofilm-forming culture on the SLIPS (top) and on superhydrophobic PTFE (bottom), scale bar $=2 \mathrm{~cm}$. c) Comparison of biofilm attachment on SLIPS based on superhydrophobic PTFE after $7 \mathrm{~d}$ of culture and a PEGyated surface after $5 \mathrm{~h}$ of culture. A) Reproduced with permission. ${ }^{[90]}$ Copyright 2012, the National Academy of Sciences. B) Comparison of the biofilm attachment of different $P$. aeruginosa strains on SLIPS prepared with porous poly (butyl methacrylate-co-ethylene dimethacrylate) (BMA-EDMA), on glass slides, and on porous BMA-EDMA after $7 \mathrm{~d}$. Reproduced with permission. ${ }^{[91]}$ Copyright 2013, the American Chemical Society. C) Number of spores of U. Linza settled after $45 \mathrm{~min}$ and $2 \mathrm{~h}$ on glass, PTFE, PEGylated gold surface, porous poly(butyl methacrylate-co-ethylene dimethacrylate) surface (BE), and BE infused with Krytox 103, Krytox 100, and Fluorinert FC 70 (abbreviated as BE103, BE100 and BE70, respectively). D) Percentage of settlement of B. Amphitrite cyprids on polystyrene, glass, PTFE, PEGlyated gold surface, BE, and on SLIPS: BE103, BE100 and BE70. C,D) Reproduced with permission. ${ }^{[22]}$ Copyright 2013, the American Chemical Society. 
of a lubricant infused PDMS coated on the inner wall of a polyurethane catheter was demonstrated. ${ }^{[33]}$ Chen et al. studied implants containing a SLIPS interface and demonstrated its resistance toward bacterial infection in vivo. It was also shown that SLIPS-modified implant significantly reduced the early host foreign body response as indicated by lower level macrophage activation correlated with a thinner fibrous capsule on SLIPS-modified implant. ${ }^{[95]}$ However, Kovalenko et al. studying silicone oil-infused PDMS confirmed the species dependence of lubricant-infused surfaces to resist bacterial attachment. The same authors also demonstrated higher number of adhered bacteria cultured under dynamic conditions in comparison to the static conditions. ${ }^{\left[{ }^{[8]}\right.}$ An excellent review about antiadhesion properties of SLIPS for medical applications including antibacterial and antithrombogenic uses of SLIPS has been recently published by Sotiri et al. ${ }^{[15]}$

SLIPS based on medically relevant materials (such as expanded polytetrafluoroethylene and perfluoroperhydrophenanthrene) was prepared, coated onto the implant surface, and then studied in a rat model. ${ }^{[95]}$ The prepared SLIPS showed good in vivo stability and could enable bacterial elimination at the implant surface, while showing low innate immune response as well as reduced inflammatory capsule formation. Suppression of occlusive thrombosis with biofunctional bloodcontacting surface is important for the practical application of medical implants. However, most of the existing biofunctional coatings either suffer from limited thrombogenic inhibition or poor durability under in vivo conditions. To enable a biofunctional coating capable of preventing thrombosis, SLIPSs were formed on a variety of commercially available medically relevant materials using a perfluorocarbon coating as the substrate and an FDA approved perfluorodecalin as lubricant. The SLIPS coated cardiopulmonary perfusion tube was assembled into arteriovenous shunts and implanted as a flowing vascular circuit in pigs. In vivo tests showed that this SLIPSfunctionalized shunts were able to suppress formation of occlusive thrombus for at least $8 \mathrm{~h}$. Considering its excellent antibiofouling performance, the reported SLIPS coating has strong potential in a number of practical medical devices. ${ }^{[99]}$ Beyond medical equipment, SLIPS have been shown to prevent fouling of membranes and help their passive recovery. ${ }^{[100]}$

Marine fouling is a longstanding problem that causes tremendous economic and environmental losses. To investigate if SLIPS could be used for antimarine fouling surfaces, the interaction between lubricant-infused porous polymer surface and marine fouling organisms was investigated. The settlement of motile spores (zoospores) of marine macroalga Ulva linza and cypris larvae of the barnacle Balanus amphitrite (cyprids) were tested (Figure 5C,D). It was observed that SLIPS based on Krytox 103 and macroporous polymethacrylate could significantly reduce the adhesion of both zoospores and cyprids. ${ }^{[22]}$ The good resistance of SLIPS against microalgae species such as Botryococcus braunii, Chlamydomonas reinhardtii, Dunaliella salina, Nannochloropsis oculata, and C. Vulgaris was also demonstrated. ${ }^{[47,101,102]}$ These results indicate the potential of SLIPS in antifouling applications in the marine environments. The antifouling properties were further demonstrated with mussel species and the SLIPS' antifouling mechanism was thoroughly investigated. ${ }^{[103]}$ Silicone oil infused polydimethylsiloxane
(i-PDMS) was able to resist mussel settlement for up to 16 weeks in field tests. The key for excellent antifouling performance of i-PDMS was that the lubricant overlayer could deceive the mechanosensing mechanism of mussels, thus preventing its adhesive behavior. Besides, the weak adhesion between the mussel's foot and i-PDMS also contributed to its antifouling properties. ${ }^{[102]}$

These results highlight the great potential of lubricantinfused coatings as a new type of material capable of preventing adhesion of eukaryotic cells, bacteria, and biofilms as well as various marine biofouler organisms. In the future, combining these properties with either dynamically controlled, responsive SLIPS or with spatially patterned SLIPS can be of both fundamental and practical interest.

\section{Other Applications and Developments}

Benefiting from its ultrasmooth surface, SLIPS were used in applications to reduce drag. ${ }^{[104]}$ In a turbulent flow, mimicking the vessel-travelling condition (flow velocity 1.0 to $3.5 \mathrm{~m} \mathrm{~s}^{-1}$ ), SLIPS revealed $10 \%$ to $16 \%$ drag reduction efficiency, while its superhydrophobic counterpart exhibited a negligible effect on reducing drag. ${ }^{[104 b, 105]}$ SLIPS could also reduce the acceleration-vibrational response within a larger frequency range than a superhydrophobic surface. In addition, the SLIPS were stable under high hydrodynamic pressure and turbulent flow fluctuations. ${ }^{[105]}$

Controlled and rapid movement of bubbles underwater is important in many technological processes such as electrochemical $\mathrm{H}_{2}$ production or $\mathrm{CO}_{2}$ capture. By introducing either hydrophilic-hydrophobic patterns or a shape gradient in the substrate of SLIPS, directional underwater bubble transport can be facilitated by the combined effect of the asymmetric driving force and low friction. ${ }^{[106,107]}$ Compared to the superhydrophobic surface, the SLIPS is particularly suitable for the underwater bubble transport because of its high pressure tolerance. The rapid transport of bubbles on this patterned surface could be ascribed to the fact that the air bubble is wrapped between the lubricant/solid interface and lubricant/water interface, which results in low adhesion of the bubbles. The integration of SLIPS into an electrochemical gas evolution device was shown by $\mathrm{Yu}$ et al. In their setup, in situ generated $\mathrm{H}_{2}$ bubbles from an $\mathrm{Al} / \mathrm{Cu}$ electrochemical erosion apparatus were directionally transported and collected via a SLIPS track. ${ }^{[108]}$

Formation of intact cell sheets is important for various applications including cell-based therapies. Juthani et al. reported the use of SLIPS based on silicone oil-infused PDMS to generate live cell sheets. ${ }^{[109]}$ The idea was to inject excess of silicone oil between the cell sheet formed on the SLIPS and the PDMS substrate in order to detach the cell layer.

Droplet collision leading to droplet interaction through a stable bilayer formed between droplets on SLIPS was demonstrated by Boreyko et al. ${ }^{[110 a]}$ Investigations on the behavior of water droplet collision on SLIPS revealed that a stable water interface phospholipid bilayer film could be formed between two colliding water droplets placed on SLIPS. By varying the viscosity of the lubricant, the amount of lubricant on SLIPS, and the size of the colliding droplets, the stability of the 
bilayer film could be tuned (Figure 2C-E). The group also demonstrated that the lifetime of droplets could be increased by changing the temperature to just above the dew point. ${ }^{[10 \mathrm{~b}]}$ Also, the process could be controlled by attaching each droplet to an agarose-coated electrode and bringing the electrodes with attached droplets close enough to each other. ${ }^{[110 b]}$ It remains to be seen whether controlled formation of droplet-droplet interfaces and networks can be accomplished through noncontact droplet actuation methods on SLIPSs. ${ }^{[31,81,110 c]}$

Slippery surface principles can be combined with other repellent surface designs. Thus, Dong et al. developed a novel slippery superoleophobic surface via combining the doubly re-entrant microtopography with SLIPS (Figure 3D). ${ }^{[111]}$ This was realized by using 3D direct laser writing to fabricate hierarchical doubly re-entrant micropillars with dedicated nanostructures on their top surfaces, followed by impregnating the nanostructured top surfaces with a hydrophobic lubricant. The slippery layer reduced the adhesion of a liquid to the pillars, while the doubly re-entrant micropillars make the surface superoleophobic compared to just oleophobic as continuous slippery surfaces.

Finally, the focus of slippery surface design is shifting from just damage tolerant slippery surfaces ${ }^{[28]}$ to effectively self-healing surfaces. ${ }^{[112,113]}$ This can be achieved by for example creation of swellable, flexible coatings as proposed by Jin et $\mathrm{al}^{\left[{ }^{[113]}\right.}$ they constructed a matrix material out of $\mathrm{Fe}_{3} \mathrm{O}_{4}$ nanoparticles armed with dopamine molecules combined with glycidyl methacrylate, polydimethylsiloxane propyl ether methacrylate, and diethylenetriamine. The matrix mechanical characteristics and surface physicochemical properties could be recovered, due to the synergistic interactions of in-built nanoparticles' intrinsic photothermal effect, reversible coordination of matrix backbone chemical building blocks, and diffused lubricating liquid. This led to a recovery from damage of $66.5 \%$ after eight cycles of cutting the matrix and actively recovering it. Alternatively, Hozumi and co-workers presented a simple slippery material, which passively recovered within 1-2 $\mathrm{h}$ after damage. It consisted of PDMS coinfused by trichloropropylsilane and low-viscosity silicone oil. While the trichloropropylsilane would spontaneously self-assemble into grass-like microfibers (named silicone micro/nanograss) on the surfaces upon exposure to ambient moisture, the silicone oil would act as a recovering agent—rapidly migrating to damaged areas of nanograss and recovering them upon damage.

\section{Summary and Outlook}

Lubricant-infused surfaces possess excellent repellency against various high- and low-surface tension liquids or complex liquid mixtures, demonstrate high stability, self-healing properties, and good optical transparency. Such surfaces exhibit very low contact angle hysteresis not only with respect to low surface tension organic solvents but also for viscous liquids, even under high-pressure conditions that are not compatible with superhydrophobic or superoleophobic states. These properties are accompanied with usually very simple design principles, facile fabrication, and diverse set of applicable substrates as well as lubricating liquids ranging from fluorinated solvents and silicone oils to mineral oils and ionic liquids. This combination of simplicity, unique properties, and variability warrants many applications in different research and industrial fields. Over the last few years, SLIPSs have already been exploited utilizing their self-cleaning, antifogging, antibiofouling, antiicing, improved heat transfer properties, or for creating cell or biofilm patterns, droplet or bubble manipulation.

There are at least two spin-off companies that are aiming to translate the SLIPS technology into commercial products ${ }^{[36]}$ and to solve real life problems, such as loss of viscous industrial liquids stuck to the walls of various containers and various types of surface biofouling for industrial and medical applications. There will definitely be more applications and commercial products in the future.

The interaction between different porous substrates and lubricants can be used to tune the surface properties. Responsive porous substrates or lubricants can be introduced into SLIPS to achieve adaptive features and dynamic control of SLIPS properties. Porous or rough substrates responsive to other stimuli, e.g., light, temperature, chemical or mechanical signals, etc., can be potentially employed to generate multifunctional stimuli-responsive SLIPSs. Another approach would be to combine responsive substrates with lubricants possessing orthogonally responsive properties to create multifunctional responsive and dynamic interfaces.

Many studies emphasized the potential of SLIPS for biomedical and antifouling applications with a few in vivo demonstrations. In the future, in vivo investigations and applications of SLIPS, integration of additional functionalities, e.g., enabling tissue integration without affecting its antifouling performance, drug delivery, dynamically controlled properties, etc., can be envisioned.

Spatially patterned lubricant-infused surfaces will also expand the scope of potential applications of SLIPS. Since the adhesion of cells, microorganisms, or biofoulers can be effectively prevented on SLIPS, substrates patterned with lubricants could be employed to create arrays of biomolecules, cells, or biofilms. ${ }^{[79,80]}$ Compared to superhydrophobic-hydrophilic micropatterns, patterned SLIPSs possess longer repellency, crucial for biological applications, as well as the other advantages, such as better pressure stability or self-healing properties. We anticipate the application of patterned SLIPS in various droplet microfluidics, sensors, cell screening and tissue engineering projects.

Despite the progress in this field, various challenges still need to be solved in this research area. Many (although not all) reported methods for preparing SLIPS are multistep processes and require the formation of intermediate porous layers, which have to be filled with a lubricant. General methods that enable the instantaneous formation of thin stable nontoxic SLIPS possessing long-term stability on typical technically relevant materials of complex shapes are required to enable further practical applications of SLIPS. Loss of the lubricant is another challenge to overcome to improve SLIPSs' performance. The overcoated lubricant layer is critical for SLIPS function, as it ultimately determines which intruding liquids can be repelled and how stable the repellency is. Careful selection of appropriate lubricants to avoid cloaking of various intruding liquids is therefore advisable. The top lubricant layer can be locked or replenished by finely tailored underlying porous substrates, for example, via 
creating "vascularized" substrates or by immobilization of the lubricant. However, even if a slippery surface is macroscopically stable, it may be unstable on the microscopic level. ${ }^{[114]}$ The lubricant film below the intruding liquid can be thinned to a thickness where molecular long-range forces come to play. ${ }^{[115]}$ Thus, if the intruding liquid possesses a high enough affinity for the surface, the lubricant film may eventually rupture. Furthermore, it has been observed that below static droplets the lubricant film may exist in a partially wetting state, even when it is present as a stable shield below the droplet in its moving form. ${ }^{[114]}$ Presence of an intact, stable oil film at all times sandwiched between the droplet and surface requires a careful choice of materials to allow for favorable van der Waals forces stabilizing the film. Miscibility of the lubricant with intruding liquids is also a limitation, necessary to be aware of. ${ }^{[19 b]}$ Although there are reports on biocompatible slippery lubricantinfused surfaces composed of, for example, chitosan and alginates, ${ }^{[116]}$ majority of SLIPS contain fluorinated lubricants. It will be necessary to minimize the use of fluorine containing chemicals and replace them with more ecofriendly and biocompatible alternatives in the future. ${ }^{[117]}$

Thus, lubricant-infused surfaces are a fascinating class of materials possessing great potential in a number of key research and industrial areas. Fundamental investigation of their properties and structure-function relationships should be an important aspect of future research in this field. This field is still in its infancy and there is a lot of room for new designs, functionalities, and applications of these exciting materials.

\section{Acknowledgements}

The work was supported by the ERC Starting Grant "DropCellArray" (337077). JL thanks National Natural Science Foundation of China for the financial support (grant No. 51502218).

\section{Conflict of Interest}

The authors declare no conflict of interest.

\section{Keywords}

antibiofouling, liquid-infused surfaces, porous surface, repellency, slippery, SLIPS

[1] M. A. Meyers, P. Y. Chen, A. Y. M. Lin, Y. Seki, Prog. Mater. Sci. 2008, 53, 1 .

[2] a) O. Z. Fisher, A. Khademhosseini, R. Langer, N. A. Peppas, Acc. Chem. Res. 2010, 43, 419; b) J. S. Mohammed, W. L. Murphy, Adv. Mater. 2009, 21, 2361; c) F. Xia, L. Jiang, Adv. Mater. 2008, 20, 2842; d) K. L. Yu, T. X. Fan, S. Lou, D. Zhang, Prog. Mater. Sci. 2013, 58, 825; e) K. S. Liu, Y. Tian, L. Jiang, Prog. Mater. Sci. 2013 , $58,503$.
[3] a) L. Jiang, Y. Zhao, J. Zhai, Angew. Chem., Int. Ed. 2004, 43, 4338; b) A. Marmur, Langmuir 2004, 20, 3517; c) L. Zhai, F. C. Cebeci, R. E. Cohen, M. F. Rubner, Nano Lett. 2004, 4, 1349.

[4] a) X. Gao, L. Jiang, Nature 2004, 432, 36; b) X. Q. Feng, X. F. Gao, Z. N. Wu, L. Jiang, Q. S. Zheng, Langmuir 2007, 23, 4892

[5] a) L. Feng, Y. A. Zhang, J. M. Xi, Y. Zhu, N. Wang, F. Xia, L. Jiang, Langmuir 2008, 24, 4114; b) B. Bhushan, E. K. Her, Langmuir 2010, 26, 8207.

[6] P. A. Zhu, T. T. Kong, X. Tang, L. Q. Wang, Nat. Commun. 2017, 8, 15823.

[7] G. D. Bixler, B. Bhushan, Nanoscale 2013, 5, 7685.

[8] A. Tuteja, W. Choi, M. L. Ma, J. M. Mabry, S. A. Mazzella, G. C. Rutledge, G. H. McKinley, R. E. Cohen, Science 2007, 318, 1618.

[9] a) T. S. Wong, S. H. Kang, S. K. Y. Tang, E. J. Smythe, B. D. Hatton, A. Grinthal, J. Aizenberg, Nature 2011, 477, 443; b) D. Quéré, Rep. Prog. Phys. 2005, 68, 2495; c) A. Lafuma, D. Quere, Europhys. Lett. 2011, 96, 5, 56001; d) J. D. Smith, R. Dhiman, S. Anand, E. Reza-Garduno, R. E. Cohen, G. H. McKinley, K. K. Varanasi, Soft Matter 2013, 9, 1772. e) M. Karakelle, J. Zdrahala Richard (Becton Dickinson and Company), EP0329041A3 1989.

[10] H. F. Bohn, W. Federle, Proc. Natl. Acad. Sci. USA 2004, 101, 14138.

[11] S. Miguel, A. Hehn, F. Bourgaud, J. Biotechnol. 2018, 265, 109.

[12] X. Yao, Y. H. Hu, A. Grinthal, T. S. Wong, L. Mahadevan, J. Aizenberg, Nat. Mater. 2013, 12, 529.

[13] a) X. Q. Wang, C. D. Gu, L. Y. Wang, J. L. Zhang, J. P. Tu, Chem. Eng. J. 2018, 343, 561; b) B. R. Solomon, S. B. Subramanyam, T. A. Farnham, K. S. Khalil, S. Anand, K. K. Varanasi, in Nonwettable Surfaces: Theory, Preparation and Applications, The Royal Society of Chemistry, London, UK 2017, Ch. 10.

[14] D. J. Preston, Z. Lu, Y. Song, Y. Zhao, K. L. Wilke, D. S. Antao, M. Louis, E. N. Wang, Sci. Rep. 2018, 8, 540.

[15] I. Sotiri, J. C. Overton, A. Waterhouse, C. Howell, Exp. Biol. Med. 2016, 241, 909.

[16] a) M. J. Kreder, J. Alvarenga, P. Kim, J. Aizenberg, Nat. Rev. Mater. 2016, 1, 15003.

[17] C. Howell, A. Grinthal, S. Sunny, M. Aizenberg, J. Aizenberg, Adv. Mater. 2018, 1802724.

[18] D. J. Preston, Y. Song, Z. M. Lu, D. S. Antao, E. N. Wang, ACS Appl. Mater. Interfaces 2017, 9, 42383.

[19] a) F. Schellenberger, J. Xie, N. Encinas, A. Hardy, M. Klapper, P. Papadopoulos, H.-J. Butt, D. Vollmer, Soft Matter 2015, 11, 7617; b) S. Sett, X. Yan, G. Barac, L. W. Bolton, N. Miljkovic, ACS Appl. Mater. Interfaces 2017, 9, 36400.

[20] Y. Wang, H. Zhang, X. Liu, Z. Zhou, J. Mater. Chem. A 2016, 4, 2524.

[21] H. L. Liu, P. C. Zhang, M. J. Liu, S. T. Wang, L. Jiang, Adv. Mater. 2013, 25, 4477.

[22] L. L. Xiao, J. S. Li, S. Mieszkin, A. Di Fino, A. S. Clare, M. E. Callow, J. A. Callow, M. Grunze, A. Rosenhahn, P. A. Levkin, ACS Appl. Mater. Interfaces 2013, 5, 10074.

[23] B. S. Lalia, S. Anand, K. K. Varanasi, R. Hashaikeh, Langmuir 2013, 29, 13081.

[24] L. Chen, A. Geissler, E. Bonaccurso, K. Zhang, ACS Appl. Mater. Interfaces 2014, 6, 6969.

[25] J. Guo, W. Fang, A. Welle, W. Feng, I. Filpponen, O. J. Rojas, P. A. Levkin, ACS Appl. Mater. Interfaces 2016, 8, 34115.

[26] a) J. L. Yong, F. Chen, Q. Yang, Y. Fang, J. L. Huo, J. Z. Zhang, X. Hou, Adv. Mater. Interfaces 2017, 4, 1700552; b) J. L. Yong, J. L. Huo, Q. Yang, F. Chen, Y. Fang, X. J. Wu, L. Liu, X. Y. Lu, J. Z. Zhang, X. Hou, Adv. Mater. Interfaces 2018, 5, 1701479.

[27] Q. Wei, C. Schlaich, S. Prevost, A. Schulz, C. Boettcher, M. Gradzielski, Z. Qi, R. Haag, C. A. Schalley, Adv. Mater. 2014, 26, 7358. 
[28] N. Vogel, R. A. Belisle, B. Hatton, T. S. Wong, J. Aizenberg, Nat. Commun. 2013, 4, 2176.

[29] K. Rykaczewski, S. Anand, S. B. Subramanyam, K. K. Varanasi, Langmuir 2013, 29, 5230.

[30] J. P. Zhang, A. Q. Wang, S. Seeger, Adv. Funct. Mater. 2014, 24, 1074.

[31] A. Eifert, D. Paulssen, S. N. Varanakkottu, T. Baier, S. Hardt, Adv. Mater. Interfaces 2014, 1, 1300138.

[32] C. Zhang, Y. F. Xia, H. Zhang, N. S. Zacharia, ACS Appl. Mater. Interfaces 2018, 10, 5892.

[33] N. MacCallum, C. Howell, P. Kim, D. Sun, R. Friedlander, J. Ranisau, O. Ahanotu, J. J. Lin, A. Vena, B. Hatton, T.-S. Wong, J. Aizenberg, ACS Biomater. Sci. Eng. 2015, 1, 43.

[34] P. Zhang, H. Chen, L. Zhang, Y. Zhang, D. Zhang, L. Jiang, J. Mater. Chem. A 2016, 4, 12212.

[35] a) K. Doll, E. Fadeeva, J. Schaeske, T. Ehmke, A. Winkel, A. Heisterkamp, B. N. Chichkov, M. Stiesch, N. S. Stumpp, ACS Appl. Mater. Interfaces 2017, 9, 9359; b) S. Zouaghi, T. Six, S. Bellayer, S. Moradi, S. G. Hatzikiriakos, T. Dargent, V. Thomy, Y. Coffinier, C. Andre, G. Delaplace, M. Jimenez, ACS Appl. Mater. Interfaces 2017, 9, 26565; c) J. Wang, K. Kato, A. P. Blois, T. S. Wong, ACS Appl. Mater. Interfaces 2016, 8, 8265.

[36] About AdaptiveSurface Technologies $®$ : https://adaptivesurface.tech/; about LiquiGlide $®$, https://liquiglide.com/about-us/ (accessed: August 2018).

[37] P. Kim, T. S. Wong, J. Alvarenga, M. J. Kreder, W. E. AdornoMartinez, J. Aizenberg, ACS Nano 2012, 6, 6569.

[38] R. Xiao, N. Miljkovic, R. Enright, E. N. Wang, Sci. Rep. 2013, 3, 1988.

[39] A. B. Tesler, P. Kim, S. Kolle, C. Howell, O. Ahanotu, J. Aizenberg, Nat. Commun. 2015, 6, 8649.

[40] H. F. Zhu, J. Hou, R. Qiu, J. Zhao, J. K. Xu, J. Appl. Polym. Sci. 2014, 131, 40184

[41] R. Qiu, Q. Zhang, P. Wang, L. N. Jiang, J. Hou, W. M. Guo, H. X. Zhang, Colloids Surf., A 2014, 453, 132.

[42] A. C. Glavan, R. V. Martinez, A. B. Subramaniam, H. J. Yoon, R. M. D. Nunes, H. Lange, M. M. Thuo, G. M. Whitesides, Adv. Funct. Mater. 2014, 24, 60.

[43] P. Juuti, J. Haapanen, C. Stenroos, H. Niemelä-Anttonen, J. Harra, H. Koivuluoto, H. Teisala, J. Lahti, M. Tuominen, J. Kuusipalo, P. Vuoristo, J. M. Mäkelä, Appl. Phys. Lett. 2017, 110, 161603.

[44] C. Howell, T. L. Vu, C. P. Johnson, X. Hou, O. Ahanotu, J. Alvarenga, D. C. Leslie, O. Uzun, A. Waterhouse, P. Kim, M. Super, M. Aizenberg, D. E. Ingber, J. Aizenberg, Chem. Mater. 2015, 27, 1792.

[45] P. Kim, M. J. Kreder, J. Alvarenga, J. Aizenberg, Nano Lett. 2013, 13, 1793.

[46] Y. Tsuge, T. Moriya, Y. Moriyama, Y. Tokura, S. Shiratori, ACS Appl. Mater. Interfaces 2017, 9, 15122.

[47] C. Howell, T. L. Vu, J. J. Lin, S. Kolle, N. Juthani, E. Watson, J. C. Weaver, J. Alvarenga, J. Aizenberg, ACS Appl. Mater. Interfaces 2014, 6, 13299

[48] V. G. Damle, A. Uppal, X. Sun, T. P. Burgin, K. Rykaczewski, Surf. Innovations 2016, 4, 102.

[49] J. X. Cui, D. Daniel, A. Grinthal, K. X. Lin, J. Aizenberg, Nat. Mater. 2015, 14, 790

[50] M. Tenjimbayashi, S. Nishioka, Y. Kobayashi, K. Kawase, J. T. Li, J. Abe, S. Shiratori, Langmuir 2018, 34, 1386.

[51] D. F. Miranda, C. Urata, B. Masheder, G. J. Dunderdale, M. Yagihashi, A. Hozumi, APL Mater. 2014, 2, 056108.

[52] Y. Galvan, K. R. Phillips, M. Haumann, P. Wasserscheid, R. Zarraga, N. Vogel, Langmuir 2018, 34, 6894.

[53] K. Greeson, K. Ghiassi, J. Alston, N. Redeker, J. Marcischak, A. Guenthner, presented at 2017 MRS Fall Meeting, Boston, MA, November 2017.
[54] K. Manabe, T. Matsubayashi, M. Tenjimbayashi, T. Moriya, Y. Tsuge, K. H. Kyung, S. Shiratori, ACS Nano 2016, 10, 9387.

[55] X. Yao, J. Ju, S. Yang, J. Wang, L. Jiang, Adv. Mater. 2014, 26, 1895.

[56] Y. J. Zheng, X. Liu, J. J. Xu, H. X. Zhao, X. H. Xiong, X. Hou, J. X. Cui, ACS Appl. Mater. Interfaces 2017, 9, 35483.

[57] D. Tian, N. Zhang, X. Zheng, G. Hou, Y. Tian, Y. Du, L. Jiang, S. X. Dou, ACS Nano 2016, 10, 6220.

[58] K. S. Khalil, S. R. Mahmoudi, N. Abu-dheir, K. K. Varanasi, Appl. Phys. Lett. 2014, 105, 041604.

[59] W. Wang, J. V. I. Timonen, A. Carlson, D.-M. Drotlef, C. T. Zhang, S. Kolle, A. Grinthal, T.-S. Wong, B. Hatton, S. H. Kang, S. Kennedy, J. Chi, R. T. Blough, M. Sitti, L. Mahadevan, J. Aizenberg, Nature 2018, 559, 77.

[60] Y. Huang, B. B. Stogin, N. Sun, J. Wang, S. K. Yang, T. S. Wong, Adv. Mater. 2017, 29, 1604641

[61] M. Y. Cao, X. Jin, Y. Peng, C. M. Yu, K. Li, K. S. Liu, L. Jiang, Adv. Mater. 2017, 29, 1606869.

[62] Z. B. Wang, L. P. Heng, L. Jiang, J. Mater. Chem. A 2018, 6, 3414.

[63] P. D. Che, L. P. Heng, L. Jiang, Adv. Funct. Mater. 2017, 27, 1606199.

[64] T. Guo, P. Che, L. Heng, L. Fan, L. Jiang, Adv. Mater. 2016, 28 , 6999.

[65] Y. F. Wang, B. T. Qian, C. L. Lai, X. W. Wang, K. K. Ma, Y. J. Guo, X. L. Zhu, B. Fei, J. H. Xin, ACS Appl. Mater. Interfaces 2017, 9, 24428.

[66] C. H. Liu, H. B. Ding, Z. Q. Wu, B. B. Gao, F. F. Fu, L. R. Shang, Z. Z. Gu, Y. J. Zhao, Adv. Funct. Mater. 2016, $26,7937$.

[67] S. Wooh, H. J. Butt, Angew. Chem., Int. Ed. 2017, 56, 4965

[68] a) C. Gao, L. Wang, Y. Lin, J. Li, Y. Liu, X. Li, S. Feng, Y. Zheng, Adv. Funct. Mater. 2018, 28, 1803072; b) Z. Wang, Y. Liu, P. Guo, L. Heng, L. Jiang, Adv. Funct. Mater. 2018, 1801310.

[69] a) D. L. Tian, Y. L. Song, L. Jiang, Chem. Soc. Rev. 2013, 42, 5184; b) E. Ueda, P. A. Levkin, Adv. Mater. 2013, 25, 1234

[70] M. Y. Cao, L. Jiang, Surf. Innovations 2016, 4, 180.

[71] R. P. Garrod, L. G. Harris, W. C. E. Schofield, J. McGettrick, L. J. Ward, D. O. H. Teare, J. P. S. Badyal, Langmuir 2007, 23, 689.

[72] a) L. Li, J. Li, X. Du, A. Welle, M. Grunze, O. Trapp, P. A. Levkin, Angew. Chem., Int. Ed. 2014, 53, 3835; b) J. S. Li, E. Ueda, A. Nallapaneni, L. X. Li, P. A. Levkin, Langmuir 2012, 28, 8286; c) F. L. Geyer, E. Ueda, U. Liebel, N. Grau, P. A. Levkin, Angew. Chem., Int. Ed. 2011, 50, 8424; d) A. I. N. A. I. Neto, C. A. Custodio, W. L. Song, J. F. Mano, Soft Matter 2011, 7, 4147; e) S. P. R. Kobaku, A. K. Kota, D. H. Lee, J. M. Mabry, A. Tuteja, Angew. Chem., Int. Ed. 2012, 51, 10109; f) E. Ueda, F. L. Geyer, V. Nedashkivska, P. A. Levkin, Lab Chip 2012, 12, 5218.

[73] a) D. Zahner, J. Abagat, F. Svec, J. M. J. Frechet, P. A. Levkin, Adv. Mater. 2011, 23, 3030; b) N. M. Oliveira, A. I. Neto, W. L. Song, J. F. Mano, Appl. Phys. Express 2010, 3, 085205.

[74] J. N. Tan, T. Alan, A. Neild, AIP Adv. 2013, 3, 022121.

[75] a) H. Notsu, W. Kubo, I. Shitanda, T. Tatsuma, J. Mater. Chem. 2005, 15, 1523; b) T. Ishizaki, N. Saito, O. Takai, Langmuir 2010, 26, 8147; c) G. Piret, E. Galopin, Y. Coffinier, R. Boukherroub, D. Legrand, C. Slomianny, Soft Matter 2011, 7, 8642.

[76] a) Y. H. Han, P. Levkin, I. Abarientos, H. W. Liu, F. Svec, J. M. J. Frechet, Anal. Chem. 2010, 82, 2520; b) S. J. Vella, P. Beattie, R. Cademartiri, A. Laromaine, A. W. Martinez, S. T. Phillips, K. A. Mirica, G. M. Whitesides, Anal. Chem. 2012, 84, 2883.

[77] C. M. Cheng, A. W. Martinez, J. L. Gong, C. R. Mace, S. T. Phillips, E. Carrilho, K. A. Mirica, G. M. Whitesides, Angew. Chem., Int. Ed. 2010, 49, 4771.

[78] J.-Y. Huang, Y.-K. Lai, F. Pan, L. Yang, H. Wang, K.-Q. Zhang, H. Fuchs, L.-F. Chi, Small 2014, 10, 4865.

[79] E. Ueda, P. A. Levkin, Adv. Healthcare Mater. 2013, 2, 1425.

[80] J. Bruchmann, I. Pini, T. S. Gill, T. Schwartz, P. A. Levkin, Adv. Healthcare Mater. 2017, 6, 1601082. 
[81] I. You, T. G. Lee, Y. S. Nam, H. Lee, ACS Nano 2014, 8, 9016.

[82] J. Hui Guan, E. Ruiz-Gutierrez, B. B. Xu, D. Wood, G. McHale, R. Ledesma-Aguilar, G. George Wells, Soft Matter 2017, 13, 3404.

[83] D. Paulssen, W. Feng, I. Pini, P. A. Levkin, Adv. Mater. Interfaces 2018, 5, 1800852.

[84] X.-C. Chen, K.-F. Ren, J. Wang, W.-X. Lei, J. Ji, ACS Appl. Mater. Interfaces 2017, 9, 1959.

[85] P. W. Wilson, W. Z. Lu, H. J. Xu, P. Kim, M. J. Kreder, J. Alvarenga, J. Aizenberg, Phys. Chem. Chem. Phys. 2013, 15, 581.

[86] S. B. Subramanyam, K. Rykaczewski, K. K. Varanasi, Langmuir 2013, 29, 13414.

[87] L. Zhu, J. Xue, Y. Y. Wang, Q. M. Chen, J. F. Ding, Q. J. Wang, ACS Appl. Mater. Interfaces 2013, 5, 4053.

[88] P. Irajizad, M. Hasnain, N. Farokhnia, S. M. Sajadi, H. Ghasemi, Nat. Commun. 2016, 7, 13395.

[89] C. Urata, G. J. Dunderdale, M. W. England, A. Hozumi, J. Mater. Chem. A 2015, 3, 12626.

[90] A. K. Epstein, T. S. Wong, R. A. Belisle, E. M. Boggs, J. Aizenberg, Proc. Natl. Acad. Sci. USA 2012, 109, 13182.

[91] J. Li, T. Kleintschek, A. Rieder, Y. Cheng, T. Baumbach, U. Obst T. Schwartz, P. A. Levkin, ACS Appl. Mater. Interfaces 2013, 5, 6704.

[92] a) P. Wang, D. Zhang, Z. Lu, S. Sun, ACS Appl. Mater. Interfaces 2016, 8, 1120; b) S. Yuan, S. Luan, S. Yan, H. Shi, J. Yin, ACS Appl. Mater. Interfaces 2015, 7, 19466.

[93] C. Wilson, J. Sandoval, D. Sabatka, E. Wilson, C. Sebest, M. Willoughby, B. Schofield, A. E. Holmes, A. L. Sutlief, Int. J. Nanotechnol. Eng. Med. 2018, 3, 7.

[94] a) M. J. Kratochvil, M. A. Welsh, U. Manna, B. J. Ortiz, H. E. Blackwell, D. M. Lynn, ACS Infect. Dis. 2016, 2, 509; b) U. Manna, N. Raman, M. A. Welsh, Y. M. Zayas-Gonzalez, H. E. Blackwell, S. P. Palecek, D. M. Lynn, Adv. Funct. Mater. 2016, 26, 3599.

[95] J. Chen, C. Howell, C. A. Haller, M. S. Patel, P. Ayala, K. A. Moravec, E. Dai, L. Liu, I. Sotiri, M. Aizenberg, J. Aizenberg, E. L. Chaikof, Biomaterials 2017, 113, 80.

[96] S. Sunny, G. Cheng, D. Daniel, P. Lo, S. Ochoa, C. Howell, N. Vogel, A. Majid, J. Aizenberg, Proc. Natl. Acad. Sci. USA 2016, 113,11676

[97] M. Badv, I. H. Jaffer, J. I. Weitz, T. F. Didar, Sci. Rep. 2017, 7, 11639.

[98] Y. Kovalenko, I. Sotiri, J. V. I. Timonen, J. C. Overton, G. Holmes, I. Aizenberg, C. Howell, Adv. Healthcare Mater. 2017, 6, 1600948

[99] D. C. Leslie, A. Waterhouse, J. B. Berthet, T. M. Valentin, A. L. Watters, A. Jain, P. Kim, B. D. Hatton, A. Nedder, K. Donovan, E. H. Super, C. Howell, C. P. Johnson, T. L. Vu, D. E. Bolgen, S. Rifai, A. R. Hansen, M. Aizenberg, M. Super, J. Aizenberg, D. E. Ingber, Nat. Biotechnol. 2014, 32, 1134.

[100] J. C. Overton, A. Weigang, C. Howell, J. Membr. Sci. 2017, 539, 257.
[101] P. Wang, D. Zhang, Z. Lu, Colloids Surf., B 2015, 136, 240.

[102] P. Wang, D. Zhang, S. Sun, T. Li, Y. Sun, ACS Appl. Mater. Interfaces 2017, 9, 972.

[103] S. Amini, S. Kolle, L. Petrone, O. Ahanotu, S. Sunny, C. N. Sutanto, S. Hoon, L. Cohen, J. C. Weaver, J. Aizenberg, N. Vogel, A. Miserez, Science 2017, 357, 668.

[104] a) M. K. Fu, I. Arenas, S. Leonardi, M. Hultmark, J. Fluid Mech. 2017, 824, 688; b) F. Veronesi, M. Raimondo, G. Boveri, C. Nicolai, E. Ciappi, L. F. Gala, presented at 2017 MRS Fall Meeting, Boston, MA, November 2017.

[105] B. J. Rosenberg, T. Van Buren, M. K. Fu, A. J. Smits, Phys. Fluids 2016, 28, 015103.

[106] a) C. H. Zhang, B. Zhang, H. Y. Ma, Z. Li, X. Xiao, Y. H. Zhang, X. Y. Cui, C. M. Yu, M. Y. Cao, L. Jiang, ACS Nano 2018, 12, 2048.

[107] X. Tang, H. R. Xiong, T. T. Kong, Y. Tian, W. D. Li, L. Q. Wang, ACS Appl. Mater. Interfaces 2018, 10, 3029.

[108] C. M. Yu, X. B. Zhu, K. Li, M. Y. Cao, L. Jiang, Adv. Funct. Mater. 2017, 27, 1701605.

[109] N. Juthani, C. Howell, H. Ledoux, I. Sotiri, S. Kelso, Y. Kovalenko, A. Tajik, T. L. Vu, J. J. Lin, A. Sutton, J. Aizenberg, Sci. Rep. 2016, 6, 26109.

[110] a) J. B. Boreyko, G. Polizos, P. G. Datskos, S. A. Sarles, C. P. Collier, Proc. Natl. Acad. Sci. USA 2014, 111, 7588; b) P. Mruetusatorn, G. Polizos, P. G. Datskos, G. Taylor, S. A. Sarles, J. B. Boreyko, D. G. Hayes, C. P. Collier, Langmuir 2015, 31, 4224; c) N. Sinn, M. T. Schür, S. Hardt, presented at 71 st Annual Meeting of the APS Division of Fluid Dynamics, Atlanta, GA, November 2018.

[111] Z. Dong, M. J. Hokkanen, B. Chang, A. Welle, Q. Zhou, R. H. A. Ras, Z. Xu, M. Wegener, P. A. Levkin, Adv. Mater. 2018, 30, 1803890.

[112] L. Wang, C. Urata, T. Sato, M. W. England, A. Hozumi, Langmuir 2017, 33, 9972.

[113] B. Jin, M. Liu, Q. Zhang, X. Zhan, F. Chen, Langmuir 2017, 33, 10340.

[114] D. Daniel, J. V. I. Timonen, R. P. Li, S. J. Velling, J. Aizenberg, Nat. Phys. 2017, 13, 1020.

[115] A. Carlson, P. Kim, G. Amberg, H. A. Stone, Europhys. Lett. 2013, 104, 34008.

[116] K. Manabe, K.-H. Kyung, S. Shiratori, ACS Appl. Mater. Interfaces 2015, 7, 4763

[117] a) C. S. Ware, T. Smith-Palmer, S. Peppou-Chapman, L. R. J. Scarratt, E. M. Humphries, D. Balzer, C. Neto, ACS Appl. Mater. Interfaces 2018, 10, 4173; b) R. Togasawa, M. Tenjimbayashi, T. Matsubayashi, T. Moriya, K. Manabe, S. Shiratori, ACS Appl. Mater. Interfaces 2018, 10, 4198.

[118] J. Chen, R. M. Dou, D. P. Cui, Q. L. Zhang, Y. F. Zhang, F. J. Xu X. Zhou, J. J. Wang, Y. L. Song, L. Jiang, ACS Appl. Mater. Interfaces 2013, 5, 4026 . 
Karlsruher Institut für Technologie

\section{Repository KITopen}

Dies ist ein Postprint/begutachtetes Manuskript.

Empfohlene Zitierung:

Li, J.; Ueda, E.; Paulssen, D.; Levkin, P. A.

Slippery Lubricant-Infused Surfaces: Properties and Emerging Applications.

2019. Advanced functional materials, 29.

doi:10.1002/adfm.201802317

Zitierung der Originalveröffentlichung:

Li, J.; Ueda, E.; Paulssen, D.; Levkin, P. A.

Slippery Lubricant-Infused Surfaces: Properties and Emerging Applications.

2019. Advanced functional materials, 29 (4), Art.Nr.: 1802317.

doi:10.1002/adfm.201802317 